PROCEEDINGS OF THE

AMERICAN MATHEMATICAL SOCIETY

Volume 139, Number 12, December 2011, Pages 4409-4418

S 0002-9939(2011)10902-X

Article electronically published on April 27, 2011

\title{
INFINITE MULTIPLICITY FOR AN INHOMOGENEOUS SUPERCRITICAL PROBLEM IN ENTIRE SPACE
}

\author{
BAISHUN LAI AND ZHIHAO GE \\ (Communicated by Walter Craig)
}

\begin{abstract}
In this paper, we will prove the existence of infinitely many positive solutions to the following supercritical problem by using the Liapunov-
\end{abstract} Schmidt reduction method and asymptotic analysis:

$$
\left\{\begin{array}{l}
\Delta u+u^{p}+f(x)=0, \quad u>0 \text { in } \mathbb{R}^{n} \\
\lim _{|x| \rightarrow \infty} u(x) \rightarrow 0
\end{array}\right.
$$

\section{INTRODUCTION AND STATEMENT OF THE MAIN RESULTS}

The purpose of this paper is to establish the existence of infinitely many positive solutions to the following inhomogeneous problem

$$
\left\{\begin{array}{l}
\Delta u+u^{p}+f(x)=0, \\
u>0 \text { in } \mathbb{R}^{n}, \lim _{|x| \rightarrow \infty} u(x)=0 .
\end{array}\right.
$$

Where $n \geq 3, p>\frac{n+2}{n-2}, \Delta=\sum_{i=1}^{n}\left(\partial^{2} / \partial x_{i}^{2}\right)$ is the Laplacian operator and $f(x) \in$ $C_{\text {loc }}^{0, \alpha}\left(\mathbb{R}^{n}\right)$ with $f \geq 0$ everywhere in $\mathbb{R}^{n}, f \not \equiv 0$.

Inhomogeneous second-order elliptic equations defined in entire space arise naturally in probability theory in the study of stochastic processes. Equation (1.1) in particular appeared recently in a paper by Tzong-Yow Lee [9] establishing limit theorems for super-brownian motion. In that paper, existence results for the equation (1.1) were obtained in the case where the inhomogeneous terms is compactly supported and is dominated by a function of the form $\frac{C}{(1+|x|)^{(n-2) p}}$ where $C(n, p)>0$ is sufficiently small. In addition to Lee [11, equation (1.1) has been studied by Pokhozhaev 12 and Egnell and Kay [10. Pokhozhaev obtained radial solutions when the inhomogeneous term is radially symmetric about the origin and satisfies certain integrability conditions. Also, Egnell and Kay worked on an equation similar to that of equation (1.1) but have a small positive parameter $\varepsilon$ as a coefficient to inhomogeneous term $f(x)$. In a recent paper [1], existence results were obtained

Received by the editors October 21, 2010.

2000 Mathematics Subject Classification. Primary 35J25, 35J20.

Key words and phrases. Critical exponents, linearized operators, supercritical problem.

The first author was supported in part by National Natural Science Foundation of China Grant 10971061 and Natural Science Foundation of Henan Province Grant 112300410054.

The second author was supported in part by National Natural Science Foundation of China Grant 10901047.

(C)2011 American Mathematical Society Reverts to public domain 28 years from publication 
for a large class of functions for the inhomogeneous term, i.e.,

$$
0 \leq f(x) \leq \frac{p-1}{\left[p\left(1+|x|^{2}\right)\right]^{\frac{p}{p-1}}} L^{p}
$$

for all $x \in \mathbb{R}^{n}$, where

$$
L=\left[\frac{2}{p-1}\left(n-2-\frac{2}{p-1}\right)\right]^{\frac{1}{p-1}} .
$$

Using sub-super solution method, Bae and Ni established the following infinite multiplicity result for the equation

$$
\left\{\begin{array}{l}
\Delta u+u^{p}+\mu f(x)=0, \quad u>0 \text { in } \mathbb{R}^{n}, \\
\lim _{|x| \rightarrow \infty} u(x) \rightarrow 0
\end{array}\right.
$$

where $\mu>0$ is a parameter.

Now, we need the following notation, which will be used throughout the paper. Set

$$
p_{c}= \begin{cases}\frac{(n-2)^{2}-4 n+4 \sqrt{n^{2}-(n-2)^{2}}}{(n-2)(n-10)} & n>10, \\ \infty & 3 \leq n \leq 10\end{cases}
$$

and

$$
\lambda_{2}=\frac{\left(n-2-\frac{4}{p-1}\right)+\sqrt{\left(n-2-\frac{4}{p-1}\right)^{2}-8\left(n-2-\frac{2}{p-1}\right)}}{2} .
$$

Now we recall Bae-Ni's result [1] as follows:

Theorem 1.1. (i) Let $p>p_{c}$. Suppose that near $\infty$

$$
\max ( \pm f(x), 0) \leq|x|^{-q_{ \pm}}
$$

where $q_{+}>n-\lambda_{2}$ and $q_{-}>n-\lambda_{2}-\frac{2}{p-1}$. Then, there exists $\mu_{*}>0$ such that for every $\mu \in\left(0, \mu_{*}\right)$, equation (1.2) possesses infinitely many solutions with asymptotic behavior $L|x|^{-m}$ at $\infty$

(ii) Let $p=p_{c}$. Then, the conclusion in (i) holds if we assume in addition that either $f$ has a compact support in $\mathbb{R}^{n}$ or $f$ does not change sign in $\mathbb{R}^{n}$.

Besides, for a more general case (i.e., $u^{p}+\mu f$ is replaced by $K(x) u^{p}+\mu f$ ), similar results are obtained; see 2, 8, 12. However, these results require $p>p_{c}, n \geq 11$. But the case of $n \leq 10$ and $\frac{n+2}{n-2}<p<p_{c}$ is open. Note that in this case, the method of sub-super solutions breaks down. In this paper, under reasonable conditions on $f$, we establish that when $p>\frac{n+1}{n-3}$, equation (1.1) has a continuum of solutions.

The main difficulty in establishing existence of equation (1.1), in addition to the noncompactness of the domain and the presence of an inhomogeneous term, is the lack of a (local) Sobolev embedding suitably fit to a weak formulation of this problem. So direct tools of the calculus of variation are not appropriate for equation (1.1).

Instead of using sub-super solution method (which limits the applicability on the exponent $p$ ), we use asymptotic analysis and the Liapunov-Schmidt reduction method to prove Theorem 1.2. This is based on the construction of a sufficiently good approximation solution. It is well known that the problem

$$
\Delta w+w^{p}=0 \text { in } \mathbb{R}^{n},
$$


where $p \geq \frac{n+2}{n-2}$, possesses a positive radially symmetric solution $w(|x|)$ which reduces to the equation

$$
w^{\prime \prime}+\frac{n-1}{r} w^{\prime}+w^{p}=0
$$

This equation can be analyzed through phase plane analysis after a transformation introduced by Fowler [9] in 1931: $v(s)=r^{\frac{2}{p-1}} w(r), r=e^{s}$, which transforms equation (1.4) into the autonomous ODE

$$
v^{\prime \prime}+\alpha v^{\prime}-\beta v+v^{p}=0
$$

where

$$
\alpha=n-2-\frac{4}{p-1}, \beta=\frac{2}{p-1}\left(n-2-\frac{2}{p-1}\right) .
$$

Since $\alpha, \beta$ are positive for $p>\frac{n+2}{n-2}$, the Hamiltonian energy

$$
E(v)=\frac{1}{2} v^{\prime 2}+\frac{1}{p+1} v^{p+1}-\frac{\beta}{2} v^{2}
$$

strictly decreases along trajectories. Using this it is easy to see the existence of a heteroclinic orbit which connects the equilibria $(0,0)$ and $\left(0, \beta^{\frac{1}{p-1}}\right)$ in the phase plane $\left(v, v^{\prime}\right)$, which correspond respectively to a saddle point and an attractor. A solution of equation (1.5) corresponding to this orbit and satisfying $v(-\infty)=$ $0, v(+\infty)=\beta^{\frac{1}{p-1}}$ and $w(r)=r^{-\frac{2}{p-1}} v(\log r)$ solves equation (1.4) and is bounded at $r=0$. Then all radial solutions of (1.3) defined in all $\mathbb{R}^{n}$ have the form

$$
w_{\lambda}(x):=\lambda^{\frac{2}{p-1}} w(\lambda|x|), \quad \lambda>0 .
$$

In what follows we denote by $w(x)$ the unique positive radial solution

$$
\Delta w+w^{p}=0 \text { in } \mathbb{R}^{n}, \quad w(0)=1 .
$$

At main order one has

$$
w(r) \sim L r^{-\frac{2}{p-1}} \text { as } r \rightarrow \infty
$$

which implies that this behavior is actually common to all solutions $w_{\lambda}(x)$.

By the change of variable $\lambda^{-\frac{2}{p-1}} u\left(\frac{x}{\lambda}\right)$ equation (1.1) becomes

$$
\Delta u+u^{p}+f_{\lambda}(x)=0, \quad \text { in } \mathbb{R}^{n} u>0, \quad \lim _{|x| \rightarrow+\infty} u(x)=0,
$$

where $f_{\lambda}(x)=\lambda^{-\frac{2 p}{p-1}} f\left(\frac{x}{\lambda}\right)$. In fact, if $f$ is assumed to satisfy the asymptotic behavior

$$
f(x)=o\left(|x|^{-\frac{2 p}{p-1}}\right) \text { as }|x| \rightarrow+\infty,
$$

then we observe that away from the origin $f_{\lambda}(x) \rightarrow 0$ as $\lambda \rightarrow 0$. Thus equation (1.1) may be regarded, away from the origin, as small perturbations of problem (1.3) when $\lambda>0$ is sufficiently small. From (1.6), we find that equation (1.1) "hides" a parameter which indexes a continuum of solutions which asymptotically vanish over compact sets.

Our main result is as follows:

Theorem 1.2. Let $p>\frac{n+1}{n-3}, n \geq 4$. Assume that

$$
f(x)=\eta(x) f_{1}(x),
$$

where $\eta(x) \in C^{\infty}\left(\mathbb{R}^{n}\right), 0 \leq \eta(x) \leq 1$,

$$
\eta(x)=0 \text { for }|x| \leq R_{1}, \eta(x)=1 \text { for }|x| \geq R_{1}+1,
$$


$\left(R_{1}>0\right.$ fixed large enough) and $f_{1}(x) \in C^{0, \alpha}\left(\mathbb{R}^{n}\right), f_{1}(x)<|x|^{-\mu}$ with $\mu>2+\frac{2}{p-1}$. Then problem (1.1) has a continuum of solutions $u_{\lambda}(x)$ (parameterized by $\lambda \leq \lambda_{0}$, where $\lambda_{0}$ is a fixed number) such that

$$
\lim _{\lambda \rightarrow 0} u_{\lambda}(x)=0
$$

uniformly in $\mathbb{R}^{n} \backslash\{0\}$. The same results holds when $\frac{n+2}{n-2}<p \leq \frac{n+1}{n-3}$ provided that $f$ is symmetric with respect to $n$ coordinate axis, namely

$$
f\left(x_{1}, \ldots, x_{i}, \ldots, x_{n}\right)=f\left(x_{1}, \ldots-x_{i}, \ldots x_{n}\right), \text { for all } i=1, \ldots, n .
$$

The idea is to consider $w(x)$ as an approximation for a solution of equation (1.1), provided that $\lambda>0$ is chosen small enough. To this end, we need to study the solvability of the operator $\Delta+p w^{p-1}$ in suitably weighted space. Recently, this issue has been studied in Davila-del Pino-Musso 4 and Davila-Pino-Musso-Wei [5].

Throughout the paper, the symbol $C$ always denotes a positive constant independent of $\lambda$, which could be changed from one line to another. Denote $A \sim B$ if and only if there exist two positive numbers $a, b$ such that $a A \leq B \leq b A$.

\section{The Solvability of Linearized operator $\Delta+p w^{p-1}$}

Our main concern in this section is to state the results concerning the existence of solutions in certain weighted spaces for

$$
\Delta \phi+p w^{p-1} \phi=h \text { in } \mathbb{R}^{n},
$$

where $w$ is the radial solution to equation (1.2) and $h$ is a known function having a specific decay at infinity. We are looking for a solution to equation (2.1) that turns out to be a perturbation of $w$. It is rather natural to require that it has a decay at most the same as that of $w$, namely $O\left(|x|^{-\frac{2}{p-1}}\right)$ as $|x| \rightarrow \infty$. Of course we would also like $\phi$ to be bounded on compact sets. As a result, we shall assume that $h$ behaves like this but with two powers subtracted; that is, $h=O\left(|x|^{-\frac{2}{p-1}-2}\right)$ at infinity.

Now, define some weighted $L^{\infty}$ norms as follows (adopted from [4, 5]):

$$
\|\phi\|_{*}=\sup _{|x| \leq 1}|x|^{\sigma}|\phi(x)|+\sup _{|x| \geq 1}|x|^{\frac{2}{p-1}}|\phi(x)|
$$

and

$$
\|h(x)\|_{* *}=\sup _{|x| \leq 1}|x|^{2+\sigma}|h(x)|+\sup _{|x| \geq 1}|x|^{2+\frac{2}{p-1}}|h(x)|,
$$

where $\sigma>0$ will be fixed later as needed.

The following lemmas and remarks on the solvability are due to Davila-del PinoMusso [4] and Davila-del Pino-Musso-Wei [5]:

Lemma 2.1. Assume that $p>\frac{n+1}{n-3}, n \geq 4$. For $0<\sigma<n-2$ there exists a constant $C>0$ such that for any $h$ with $\|h(x)\|_{* *}<\infty$, equation (2.1) has a solution $\phi=T(h)$ such that $T$ defines a linear map and

$$
\|T(h)\|_{*} \leq C\|h\|_{* *} .
$$

For the sake of completeness, we give the main idea of the proof of Lemma 2.1 as follows (for the details, see [4, 5]):

Let $\Theta_{k}, k \geq 0$ be the eigenfunction of the Laplace-Beltrami operator $-\Delta_{s^{n-1}}$ on the sphere $S^{n-1}$ with eigenvalues $\lambda_{k}$ repeated according to their multiplicity, normalized so that they constitute an orthonormal system in $L^{2}\left(S^{n-1}\right)$. Let $\Theta_{0}$ 
be a positive constant associated to the eigenvalue 0 and $\Theta_{i}, 1 \leq i \leq n$, which is an appropriate multiple of $\frac{x_{i}}{|x|}$ which has eigenvalue $\lambda_{i}=n-1,1 \leq i \leq n$. We repeat eigenvalues according to their multiplicity and we arrange them in an nondecreasing sequence. We recall that the set of eigenvalues is given by $\{j(n-2+j) \mid j \geq$ $0\}$. We write $h$ as

$$
h(x)=\Sigma_{k=0}^{k=+\infty} h_{k} \Theta_{k}(\theta)
$$

and look for a solution $\phi$ to $(2.1)$ in the form

$$
\phi(x)=\Sigma_{k=0}^{k=+\infty} \phi_{k} \Theta_{k}(\theta)
$$

Then

$$
\phi_{k}^{\prime \prime}+\frac{n-1}{r} \phi^{\prime}+\left(p w^{p-1}-\frac{\lambda_{k}}{r^{2}}\right) \phi=h_{k} .
$$

Equation (2.4) can be solved for each $k$ separately:

a: If $k=0$ and $p>\frac{n+2}{n-2},\left\|h_{0}\right\|_{* *}<+\infty$, then (2.4) has a solution $\phi_{0}$ which depends linearly on $h_{0}$ and satisfies

$$
\left\|\phi_{0}\right\|_{*} \leq C\left\|h_{0}\right\|_{* *}
$$

Indeed in this case this solution is defined using the variation of parameters formula

$$
\phi_{0}(r):=z_{1,0}(r) \int_{1}^{r} z_{2,0} h_{0} s^{n-1} d s-z_{2,0}(r) \int_{0}^{r} z_{1,0} h_{0} s^{n-1} d s,
$$

where $z_{1,0}, z_{2,0}$ are two special linearly independent solutions to (2.4) with $k=0$ and $h_{0}=0$. More precisely, we take $z_{1,0}=r w^{\prime}+\frac{2}{p-1} w$ and a second, linearly independent solution $z_{2,0}$ can be found in any interval where $z_{1,0}$ does not vanish as

$$
z_{2,0}=z_{1,0} \int z_{1,0}(r)^{-2} r^{1-N} d r
$$

Linearization shows that

$$
z_{j, 0}(r)=O\left(r^{-\frac{n-2}{2}}\right) \text { as } r \rightarrow+\infty, \quad j=1,2
$$

while

$$
z_{2,0} \sim r^{2-n} \text { near } r=0
$$

Using this and (2.6), we can easily get estimate (2.5).

b: If $k=1, n \geq 4$ and $p>\frac{n+1}{n-3},\left\|h_{1}\right\|_{* *}<+\infty$, then we have

$$
\left\|\phi_{1}\right\|_{*} \leq C\left\|h_{1}\right\|_{* *} \text {. }
$$

In this case, we have that the positive function $z_{1}:=-w^{\prime}(r)>0$ in $(0,+\infty)$ solves (2.4) with $k=1$ and $h_{1}=0$. Using this, we then define $\phi_{1}(r)$ as

$$
\phi_{1}(r)=-z_{1}(r) \int_{1}^{r} z_{1}(s)^{-2} s^{1-n} d s \int_{0}^{s} z_{1}(\tau) h_{1}(\tau) \tau^{n-1}
$$

Using this formula and by a simple computer, estimate (2.7) is easily obtained.

c: Let $k \geq 2$ and $p>\frac{n+2}{n-2}$. If $\left\|h_{k}\right\|_{* *}<+\infty$ (2.4) has a unique solution $\phi_{k}$ with $\left\|\phi_{k}\right\|_{*}<+\infty$ and there exists $C_{k}>0$ such that

$$
\left\|\phi_{k}\right\|_{*} \leq C\left\|h_{k}\right\|_{* *}
$$

this case is simpler because the operator

$$
L_{k} \phi=\phi^{\prime \prime}+\frac{n-1}{r} \phi^{\prime}+\left(p w^{p-1}-\frac{\lambda_{k}}{r^{2}}\right) \phi
$$


satisfies the maximum principle in any interval of the form $\left(\delta, \frac{1}{\delta}\right), \delta>0$. Indeed let $z=-w^{\prime}$ so that $z>0$ in $(0,+\infty)$ and it is a supersolution, because

$$
L_{k} z=\frac{n-1-\lambda_{k}}{r^{2}} z<0 \text { in }(0,+\infty),
$$

since $\lambda_{k} \geq 2 n$ for $k \geq n$. We construct a supersolution $\psi$ of the form

$$
\psi=C_{1} z+v, \quad v(r)=\frac{1}{r^{\sigma}+r^{\frac{2}{p-1}}} .
$$

Choosing $C_{1}$ sufficiently large, we can check that

$$
L_{k} \psi \leq-c \min \left(r^{-\sigma-2}, r^{-\frac{2}{p-1}-2}\right) \text { in }(0,+\infty) .
$$

Using this, we can easily obtain (2.9).

The previous construction and (2.5), (2.7) and (2.9) imply that given an integer $m>0$, if $\|h\|<+\infty$ satisfies $h_{k} \equiv 0 \quad \forall k \geq m$, there exists a solution $\phi$ to (3.1) that depends linearly with respect to $h$ and moreover

$$
\|\phi\|_{*} \leq C_{m}\|h\|_{* *}
$$

where $C_{m}$ may depend on only $m$. Then by using a blow-up argument, we can show that $C_{m}$ can be chosen independently of $m$. For a detailed proof, we refer the interested reader to [5, 6].

Remark 2.2. From the above proof, we know the operator $T$ in Lemma 2.1 is constructed "by hand" by decomposing $h$ and $\phi$ into sums of spherical harmonics where the coefficients are radial functions. The nice property is of course that $w$ is radial, so the problem decouples into an infinite collection of ODEs.

Remark 2.3. If $p \geq \frac{n+2}{n-2}$, linearized operator $\Delta+p w^{p-1}$ has a kernel, i.e., $\left\{\frac{\partial w}{\partial x_{1}}, i=\right.$ $1, \ldots, n\}$, in the general (usual) Sobolev space. However, under suitable weighted space, the linearized operator $\Delta+p w^{p-1}$ is invertible; i.e., the kernel is 0 .

\section{The Proof of Theorem 1.2}

Let $p>\frac{n+1}{n-3}$. We will prove Theorem 1.2 in this section. The main idea is to use Lemma 2.1 and a contraction mapping principle.

We look for a solution of equation (1.5) of the form $u=w+\phi$, which yields the following equation for $\phi$ :

$$
\Delta \phi+p w^{p-1} \phi=N(\phi)-f_{\lambda}(x)
$$

where

$$
N(\phi)=-(w+\phi)^{p}+w^{p}+p w^{p-1} \phi .
$$

Using the operator $T$ defined in Lemma 2.1, we are led to solving the fixed point problem

$$
\phi=T\left(N(\phi)-f_{\lambda}(x)\right) .
$$

Firstly let us estimate $\|N(\phi)\|_{* *, \lambda}$ depending on whether $p \geq 2$ or $p<2$.

Case $p \geq 2$. In this case, we observe that

$$
|N(\phi)| \leq C\left(w^{p-2} \phi^{2}+|\phi|^{p}\right) .
$$

Let us work with $0<\sigma \leq \frac{2}{p-1}$. Since

$$
|\phi(x)| \leq C|x|^{-\frac{2}{p-1}}\|\phi\|_{*}, \text { for all }|x| \geq 1,
$$


and

$$
w(x) \leq C(1+|x|)^{-\frac{2}{p-1}}, \text { for all } x \in \mathbb{R}^{n},
$$

we have on one hand

$$
\sup _{|x| \geq 1}|x|^{2+\frac{2}{p-1}} w^{p-2}|\phi|^{2} \leq C\|\phi\|^{2} .
$$

On the other hand,

$$
|\phi| \leq C|x|^{-\sigma}\|\phi\|_{*}, \text { for all }|x| \leq 1,
$$

and therefore, we obtain

$$
\sup _{|x| \leq 1}|x|^{2+\sigma} w(x)^{p-2}|\phi|^{2} \leq\|\phi\|_{*}^{2} \sup _{|x| \leq 1}|x|^{2-\sigma}=C\|\phi\|_{*}^{2} .
$$

From (3.3) and (3.4) it follows that

$$
\left\|w^{p-2} \phi^{2}\right\|_{* *} \leq C\|\phi\|_{*}^{2} .
$$

To estimate $\left\||\phi|^{p}\right\|_{* *}$ we compute

$$
\sup _{|x| \leq 1}|x|^{2+\sigma}|\phi(x)|^{p} \leq C\|\phi\|_{*}^{p} .
$$

Similarly

$$
\sup _{|x| \geq 1}|x|^{2+\frac{2}{p-1}}|\phi|^{p} \leq\|\phi\|_{*}^{p} .
$$

From (3.6) and (3.7) it follows that

$$
\left\||\phi|^{p}\right\|_{* *} \leq C\|\phi\|_{*}^{p} .
$$

By (3.5) and (3.8) we have

$$
\|N(\phi)\|_{* *} \leq C\left(\|\phi\|_{*}^{2}+\|\phi\|_{*}^{p}\right) .
$$

Case $p<2$. In this case $|N(\phi)| \leq C|\phi|^{p}$, and hence, if $0<\sigma \leq \frac{2}{p-1}$,

$$
\sup _{|x| \leq 1}|x|^{2+\sigma}|\phi(x)|^{p} \leq C\|\phi\|_{*}^{p} .
$$

Similarly

$$
\sup _{|x| \geq 1}|x|^{2+\frac{2}{p-1}}|\phi|^{p} \leq\|\phi\|_{*}^{p} .
$$

From (3.10) and (3.11) it follows that for any $1<p<2$ and $0<\sigma \leq \frac{2}{p-1}$,

$$
\|N(\phi)\|_{* *} \leq C\|\phi\|_{*}^{p} .
$$

From (3.9) and (3.12) we have

$$
\|N(\phi)\|_{* *} \leq C\left(\|\phi\|_{*}^{2}+\|\phi\|_{*}^{p}\right) .
$$


Now, we estimate $\left\|f_{\lambda}(x)\right\|_{* *}$ as follows:

(3.14) $\sup _{|x| \leq 1}|x|^{2+\sigma}\left|f_{\lambda}(x)\right|=\sup _{\lambda R_{1}<|x| \leq 1}|x|^{2+\sigma}\left|f_{\lambda}(x)\right| \leq R_{1}^{2+\sigma-\mu} \rightarrow 0$ as $R_{1} \rightarrow+\infty$,

provided that $\sigma=\frac{2}{p-1}$.

Similarly,

$$
\sup _{|x| \geq 1}|x|^{2+\frac{2}{p-1}}\left|f_{\lambda}(x)\right|=o(\lambda) .
$$

So we have, as $\lambda \rightarrow 0$,

$$
\left\|f_{\lambda}(x)\right\|_{* *}=\sup _{|x| \leq 1}|x|^{2+\sigma}\left|f_{\lambda}(x)\right|+\sup _{|x| \geq 1}|x|^{2+\frac{2}{p-1}}\left|f_{\lambda}(x)\right| \leq R_{1}^{\mu-2-\sigma} .
$$

We have already observed that $u=w+\phi$ is a solution of (1.5) if $\phi$ satisfies equation (3.2). Consider the set

$$
F=\left\{\phi \in L^{\infty} /\|\phi\|_{*} \leq \rho\right\}
$$

where $\rho \in(0,1)$ is to be chosen (suitably small) and the operator is

$$
\hbar(\phi)=T\left(N(\phi)-f_{\lambda}(x)\right) .
$$

We now prove that $\hbar$ has a fixed point in $F$. For $\phi \in F$ we have

$$
\begin{aligned}
\|\hbar(\phi)\|_{*} & \leq C\|N(\phi)\|_{* *}+C\left\|f_{\lambda}\right\|_{* *} \\
& \leq C\left(\|\phi\|_{*}^{2}+\lambda^{-2}\|\phi\|_{*}^{p}+R_{1}^{\mu-2-\sigma}\right)
\end{aligned}
$$

by (3.9) and (3.10) if $\sigma=\frac{2}{p-1}$. Then we have

$$
\|\hbar(\phi)\|_{*, \lambda} \leq C\left(\rho^{2}+\rho^{p}+R_{1}^{\mu-2-\sigma}\right) \leq \rho
$$

if we choose $\rho$ small enough and $R_{1}$ large enough. Hence $\hbar(F) \subset F$.

Now we show that $\hbar$ is a contraction mapping in $F$. Let us take $\phi_{1}, \phi_{2}$ in $F$; then

$$
\left\|\hbar\left(\phi_{1}\right)-\hbar\left(\phi_{2}\right)\right\|_{*} \leq C\left\|N\left(\phi_{1}\right)-N\left(\phi_{2}\right)\right\|_{* *} .
$$

Write

$$
N\left(\phi_{1}\right)-N\left(\phi_{2}\right)=D_{\phi} N(\bar{\phi})\left(\phi_{1}-\phi_{2}\right),
$$

where $\bar{\phi}$ lies in the segment joining $\phi_{1}$ and $\phi_{2}$.

For $|x| \leq 1$,

$$
|x|^{2+\sigma}\left|N\left(\phi_{1}\right)-N\left(\phi_{2}\right)\right| \leq|x|^{2}\left|D_{\phi} N(\bar{\phi})\right||| \phi_{1}-\phi_{2} \|_{*},
$$

while, for $|x| \geq 1$,

$$
|x|^{2+\frac{2}{p-1}}\left|N\left(\phi_{1}\right)-N\left(\phi_{2}\right)\right| \leq|x|^{2}\left|D_{\phi} N(\bar{\phi})\right||| \phi_{1}-\phi_{2} \|_{*} .
$$

Then we have

$$
\left\|N\left(\phi_{1}\right)-N\left(\phi_{2}\right)\right\|_{* *} \leq C \sup _{x}|x|^{2}\left|D_{\phi} N(\bar{\phi})\right|\left\|\phi_{1}-\phi_{2}\right\|_{*} .
$$

Directly from the definition of $N$, we compute

$$
D_{\phi} N(\bar{\phi})=p\left[(w+\bar{\phi})^{p-1}-w^{p-1}\right] .
$$

Thus

$$
\left|D_{\phi} N(\bar{\phi})\right| \leq C\left(w^{p-2}|\bar{\phi}|+|\bar{\phi}|^{p-1}\right) .
$$

For all $x$ we have

$$
|x|^{2} w^{p-2}|\bar{\phi}| \leq C\left(\left\|\phi_{1}\right\|_{*}+\left\|\phi_{2}\right\|_{*}\right) \leq C \rho .
$$


Similarly, for all $x$

$$
|x|^{2}|\bar{\phi}|^{p-1} \leq C\left(\left\|\phi_{1}\right\|_{*}^{p-1}+\left\|\phi_{2}\right\|_{*}^{p-1}\right) \leq C \rho^{p-1} .
$$

Estimates (3.13)-(3.15) show that

$$
\sup _{x}|x|^{2}\left|D_{\phi} N(\bar{\phi})\right| \leq C\left(\rho+\rho^{p-1}\right) .
$$

Gathering relations (3.11), (3.12), (3.16) we conclude that $\hbar$ is a contraction mapping in $F$, and hence a fixed point in this region indeed exists. So $w+\phi_{\lambda}$ is the solution of

$$
\Delta u+u^{p}+f_{\lambda}(x)=0, \quad \text { in } \mathbb{R}^{n} u>0, \quad \lim _{|x| \rightarrow+\infty} u(x)=0,
$$

and

$$
\phi_{\lambda}(x) \leq C \quad \text { for all } \quad x \in \mathbb{R}^{n} \backslash\{0\} .
$$

Thus $u_{\lambda}(x)=\lambda^{\frac{2}{p-1}}\left(w(\lambda x)+\phi_{\lambda}(\lambda x)\right)$ is a continuum of (1.1) and

$$
\lim _{\lambda \rightarrow 0} u_{\lambda}(x)=0
$$

uniformly in $\mathbb{R}^{n} \backslash\{0\}$. This finishes the proof of Theorem 1.2.

To conclude, in this paper, instead of using the sub-super solution method (which limits the applicability on the exponent $p$ ), we use asymptotic analysis and the Liapunov-Schmidt reduction method to solve an open problem.

\section{ACKNOWLEDGEMENTS}

The first author is greatly indebted to his advisor, Professor Yi Li, for many useful discussions, constant support, and encouragement. He would also like to thank the anonymous referee for a careful reading of this paper.

\section{REFERENCES}

1. S. Bae and W-M.Ni, Existence and infinite multiplicity for an inhomogeneous semilinear elliptic equation on $\mathbb{R}^{n}$, Math. Ann. 320 (2001), 191-210. MR1835068(2002i:35052)

2. S. Bae, T.-K. Chang and D.-H. Pank, Infinite multiplicity of positive entire solutions for a semilinear elliptic equation, J. Differential Equations 181 (2002), 367-387. MR 1907146 (2003i:35076)

3. G. Bernard, An inhomogeneous semilinear equation in entire space, J. Differential Equations 125 (1996), 184-214. MR1376065 (97d:35050)

4. J. Dávila, M. del Pino, M. Musso and J. Wei, Standing waves for supercritical nonlinear Schrödinger equations, J. Differential Equations 236 (2007), 164-198. MR2319924 (2009b:35389)

5. J. Dávila, M. del Pino, M. Musso and J. Wei, Fast and slow decay solutions of supercritical problems in exterior domains, Calculus of Variations and PDE 32 (2008), 453-480. MR 2402919 (2009b:35140)

6. M. del Pino, Supercritical elliptic problems from a perturbation viewpoint, Discrete and Continous Dynamical Systems 21 (2008), 69-89. MR2379457 (2009b:35130)

7. M. del Pino and J. Wei, Supercritical elliptic problems in domains with small holes, Ann. Inst. H. Poincaré Anal. Non Lineaire 24 (2007), 507-520. MR.2334989 (2008j:35077)

8. Y.-B. Deng, Y. Li and F. Yang, On the stability of the positive steady states for a nonhomogeneous semilinear Cauchy problem, J. Differential Equations 228 (2006), 507-529. MR2289543 $(2008 \mathrm{~m}: 35172)$

9. R. H. Fowler, Further studies on Emden's and similar differential equations, Quart. J. Math. 2 (1931), 259-288.

10. H. Egnell and I. Kaj, Positive global solutions of a nonhomogeneous semilinear elliptic equation, J. Math. Pures Appl. (9) 70, No. 3 (1991), 345-367. MR:1113816 (92m:35078) 
11. T.-Y. Lee, Some limit theorems for super-Brownian motion and semilinear differential equations, Ann. Probab. 21, No. 2 (1993), 979-995. MR1217576 (94b:60038)

12. S. I. Pokhozhaev, On the solvability of an elliptic problem in Rn with a supercritical index of nonlinearity, Soviet Math. Dokl. 42, No. 1 (1991), 215-219. MR1080040 (91j:35100)

13. C.-F. Gui, Positive entire solutions of equation $\Delta u+F(x, u)=0$, J. Differential Equations 99 (1992), 245-280. MR:1184056 (93h:35065)

Institute of Contemporary Mathematics, Henan University, Kaifeng 475004, People's Republic of China

Current address: School of Mathematics and Information Science, Henan University, Kaifeng 475004, People's Republic of China

E-mail address: laibaishun@henu.edu.cn

School of Mathematics and Information Science, Henan University, Kaifeng 475004, People's Republic of China

E-mail address: zhihaoge@gmail.com 